\title{
Borda, Cancellation and Belief Merging
}

\author{
Patricia Everaere $^{1}$, Chouaib Fellah ${ }^{2}$, Sébastien Konieczny ${ }^{2}$, Ramón Pino Pérez ${ }^{2}$ \\ ${ }^{1}$ Univ. Lille, CNRS, Centrale Lille, UMR 9189, CRIStAL, F-59000 Lille, France \\ ${ }^{2}$ CRIL - CNRS - Université d'Artois - France \\ patricia.everaere-caillier@univ-lille.fr, \{fellah,konieczny,pinoperez $\} @$ cril.fr
}

\begin{abstract}
In this work, we explore the links between the Borda voting rule and belief merging operators. More precisely, we define two families of merging operators inspired by the definition of the Borda voting rule. We also introduce a notion of cancellation in belief merging, inspired by the axiomatization of the Borda voting rule proposed by Young. This allows us to provide a characterization of the drastic merging operator.
\end{abstract}

\section{Introduction}

Belief merging operators aim at synthesizing a set of conflicting belief bases in order to form a coherent view of the world. To this aim, these operators benefit from the complementarity of the belief bases, which allows to generate new pieces of information that are distributed in the belief bases, while solving the logical conflicts between these bases.

Belief merging (Revesz 1997; Lin and Mendelzon 1998; Liberatore and Schaerf 1998; Konieczny and Pino Pérez 1998; Konieczny and Pino Pérez 2002; Konieczny and Pino Pérez 2011) can be considered as the intersection of two research topics. The first one is belief revision (Alchourrón, Gärdenfors, and Makinson 1985; Gärdenfors 1988; Katsuno and Mendelzon 1991; Fermé and Hansson 2011), where the problem is to correct the beliefs of the agent by a (more reliable) piece of information. In both revision and merging the problem is to find the most plausible information given the input. The difference is that for revision the input is a single belief base, whereas for merging it is a set of such bases. So the principles governing these two problems are closely related. The second research topic is group decision as studied in social choice theory (Arrow 1963; Arrow, Sen, and Suzumura 2002), and especially in voting methods. The aim of a voting method is to define a social preference (a preference for the whole group) from a set of individual preferences given by the individuals. Similarly belief merging aims at defining the "beliefs of the group" from the beliefs provided by several sources. So social choice (particularly voting methods) and belief merging share concern about how to faithfully take into account the set of inputs (preferences for vote, beliefs for merging).

Formal links between belief merging and belief revision are well known. For instance it is easy to show that belief revision can be considered as a special case of belief merging when there is a single belief base in the profile (Konieczny and Pino Pérez 2002).

Links between belief merging and social choice theory have also been investigated. For instance in (Konieczny and Pino Pérez 2005) there is a comparison between social choice functions and belief merging operators. Some questions coming from social choice and voting methods have also been investigated in the context of belief merging. For instance, (Everaere, Konieczny, and Marquis 2007) studies the manipulation issue for merging operators. (Everaere, Konieczny, and Marquis 2010b) investigates the problem of finding the truth (truth-tracking) and a generalization of Condorcet's Jury Theorem for belief merging operators is given. Some egalitarian belief merging operators were proposed using standard techniques coming from social choice (Everaere, Konieczny, and Marquis 2014a). A generalisation of Arrow's impossibility Theorem in the framework of belief merging is given in (Mata Díaz and Pino Pérez 2017). Finally, some works on judgment aggregation and its link with belief merging have been done (Everaere, Konieczny, and Marquis 2015). Judgment aggregation can be seen as an intermediate issue between voting and belief merging. As in belief merging, judgment aggregation (List and Pettit 2002; List and Puppe 2009; Pigozzi 2006; Everaere, Konieczny, and Marquis 2014b; Lang et al. 2017) works from logical formulae, and as in (multiple) vote (and contrarily to merging), there is an agenda (a set of formulae) on which the group has to decide.

In this work we want to investigate a more direct and more technical link between some merging operators and a voting method. In fact a wide class of belief merging operators is the class of distance-based merging operators (Konieczny, Lang, and Marquis 2004; Konieczny and Pino Pérez 2011). These operators use a distance in order to generate an evaluation of the plausibility of each possible world with respect to each belief base from the input profile, and then they use an aggregation function in order to obtain a global evaluation of the plausibility of each possible world.

Several aggregation functions can be considered in order to give rise to sensible operators, like the sum (Revesz 1997; Lin and Mendelzon 1998), the leximax (Konieczny and Pino Pérez 2002), the sum of the $\mathrm{n}^{\text {th }}$ powers (Konieczny and Pino Pérez 2005), the leximin (Everaere, Konieczny, and Marquis 2010a), etc. 
When using the sum as aggregation function, the corresponding operators are quite close to the Borda voting rule (Borda 1781; Young 1974; Merlin 2003). In both cases the information provided by the individual sources generates a score (numerical evaluation) for each alternative, that are then aggregated using the sum.

There are some differences between the two processes. For the Borda voting rule the source provides a (linear) order. For belief merging, the source provides a belief base. The distance used to define the merging operator may produce a corresponding ordering, which is, in fact, a total preorder (we will see that this has an impact on the formal results).

But the similarity is large enough to warrant some deeper investigations. In particular, there are some interesting formal results on the Borda voting rule which we want to investigate in the belief merging context. This allows us to define two new families of belief merging operators and a characterization result.

Basically, there are two definitions for the Borda voting rule. These two definitions are equivalent when linear orders are considered. But it is no longer the case on general preorders. So we obtain two different definitions, that, applied to belief merging, give us new merging operators.

Another interesting formal result is that the Borda voting rule is characterized by a cancellation property (Young 1974). This property is really about the orders, and cannot be directly translated to belief merging. But this idea can give us interesting counterparts. We investigate this class of cancellation properties. And we give a characterization of a belief merging operator using one of these properties.

The plan of the paper is as follows. The next Section provides the required notions and notations for the paper. Then in Section 3 we recall the definition of IC merging operators, the representation theorem in terms of plausibility preorders, and the definition of distance-based merging operators. In Section 4 we give the two definitions of the Borda voting rule, we show that they are not equivalent when preorders are used, and we give the cancellation property. In Section 5 we give the definition of the two families of Borda-like merging operators. These operators need a function that associates a preorder to each belief base. This can be provided, for instance, by an IC merging operator or by a belief revision operator. Then in Section 7 we investigate the properties of these two families of operators. And in Section 8 we investigate the cancellation properties and we provide a characterization of the $\Delta^{d_{D}, f}$ operator. We conclude in Section 9.

\section{Preliminaries}

We consider a propositional language $\mathcal{L}$ defined from a finite set of propositional variables $\mathcal{P}$ and the standard connectives. An interpretation $\omega$ is a total function from $\mathcal{P}$ to $\{0,1\}$. The set of all interpretations is noted $\Omega$. An interpretation $\omega$ is a model of a formula $\varphi \in \mathcal{L}$ if and only if it makes it true in the usual truth functional way. $[[\varphi]]$ denotes the set of models of the formula $\varphi$, i.e., $[[\varphi]=\{\omega \in \Omega: \omega \mid=\varphi\}$. When $M$ is a set of models, we denote by $\varphi_{M}$ a formula such that $\left[\left[\varphi_{M}\right]\right]=M$.
A profile $E$ is a vector of formulae $E=\left(\varphi_{i_{1}}, \ldots, \varphi_{i_{n}}\right)$ where each $i_{j}$ represents an agent/individual/source (hence different agents/individuals/sources are allowed to exhibit identical formulae), so it represents a group of $n$ agents. We denote by $\mathcal{E}$ the set of profiles. $\bigwedge E$ represents the conjunction of formulae of $E=\left(\varphi_{i_{1}}, \ldots, \varphi_{i_{n}}\right)$, i.e., $\bigwedge E=\varphi_{i_{1}} \wedge$ $\ldots \wedge \varphi_{i_{n}}$. A profile $E$ is said to be consistent if and only if $\bigwedge E$ is consistent. Two profiles $E_{1}=\left(\varphi_{i_{1}}, \ldots, \varphi_{i_{n}}\right), E_{2}=$ $\left(\varphi_{j_{1}}, \ldots, \varphi_{j_{n}}\right)$ are equivalent, denoted $E_{1} \equiv E_{2}$, if there is a bijective function $\sigma$ from $\left\{i_{1}, \ldots, i_{n}\right\}$ onto $\left\{j_{1}, \ldots, j_{n}\right\}$ such that for every $i_{k} \in\left\{i_{1}, \ldots, i_{n}\right\}, \varphi_{i_{k}} \equiv \varphi_{\sigma\left(i_{k}\right)}$. The concatenation of profiles ${ }^{1}$ is noted $\sqcup$. By abuse of notation we will write $\varphi \sqcup E$ instead of $(\varphi) \sqcup E$. We denote by $E^{n}$ the profile $E$ "concatenated with itself $n$ times". More precisely $E^{n}=E_{1} \sqcup \ldots \sqcup E_{n}$ where each $E_{i}$ is equivalent to $E$. The notation $\neg E$ represents the profile composed of the negations of the belief bases of the profile $E=\left(\varphi_{i_{1}}, \ldots, \varphi_{i_{n}}\right)$, i.e. $\neg E=\left(\neg \varphi_{i_{1}}, \ldots, \neg \varphi_{i_{n}}\right)$.

A total preorder over a set $X$ is a binary relation $\preceq$ that is reflexive, transitive and total, $\prec$ denotes the associated strict relation defined by $\omega \prec \omega^{\prime}$ if and only if $\omega \preceq \omega^{\prime}$ and $\omega^{\prime} \npreceq \omega$. Let $\preceq$ be a total preorder on $X$, and $B \subseteq X$, then $\min (B, \preceq)=\{b \in B: \nexists x \in B, x \prec b\}$.

If $A$ is a set, we denote $|A|$ the cardinality of $A$. The symbol $\subseteq$ will denote set containment and $\subset$ strict set containment, i.e., $A \subset B$ if and only if $A \subseteq B$ and $A \neq B$.

When $\preceq$ is a total preorder over $X$, the canonical ranking function $r_{\preceq}: X \rightarrow \mathbb{N}$, associated to $\preceq$ is defined by putting $r_{\preceq}(x)$ as the maximum of integers $k$ such that there exists $x_{0}, x_{1}, \ldots, x_{k}$ in $X$ with $x_{k}=x$ and $x_{i} \prec x_{i+1}$ for $i=$ $0, \ldots, k-1$.

Suppose $X=\left\{x_{1}, \ldots, x_{m}\right\}$. Given a vector $P=$ $\left(\preceq_{1}, \ldots, \preceq_{n}\right)$ of total preorders, we define $\pi_{i j}(P)$, for any $i, j \in\{1, \ldots, m\}$, by $\pi_{i j}(P)=\left|\left\{1 \leq k \leq n: x_{i} \prec_{k} x_{j}\right\}\right|$. $\pi_{i j}(P)$ represents the number of bases in the vector $P$ which prefer $x_{i}$ to $x_{j}$. Note that $\pi_{i i}(P)=0$ for any $i \in\{1, \ldots, m\}$, and that $\forall i, j \in\{1, \ldots, m\}, \pi_{i j}(P) \geq 0$.

The merging operators we will consider are functions from the set of profiles and the set of propositional formulae (that will represent integrity constraints noted $\mu$ ) to the set of formulae, i.e. $\Delta: \mathcal{E} \times \mathcal{L} \mapsto \mathcal{L}$. We will use the notation $\Delta_{\mu}(E)$ instead of $\Delta(E, \mu)$. We write also $\Delta(E)$ instead of $\Delta_{\top}(E)$ where $T$ is the tautological formula.

\section{IC Merging}

Let us recall in this Section the postulates for IC merging, the representation theorem, and the definition of distancebased operators (Konieczny and Pino Pérez 2002).

Definition 1. A merging operator $\Delta$ is called an IC merging operator if it satisfies postulates (ICO-IC8). An IC merging operator $\Delta$ is called a majority merging operator if it satisfies postulate (Maj).

(IC0) $\Delta_{\mu}(E) \models \mu$

(IC1) If $\mu$ is consistent, then $\Delta_{\mu}(E)$ is consistent

${ }^{1} \mathrm{We}$ assume that the profiles to be concatenated have disjoint sets of agents. 
(IC2) If $\wedge E \wedge \mu$ is consistent, then $\Delta_{\mu}(E) \equiv \wedge E \wedge \mu$

(IC3) If $E_{1} \equiv E_{2}$ and $\mu_{1} \equiv \mu_{2}$, then $\Delta_{\mu_{1}}\left(E_{1}\right) \equiv \Delta_{\mu_{2}}\left(E_{2}\right)$

(IC4) If $\varphi_{1} \models \mu, \varphi_{2} \models \mu$ and $\Delta_{\mu}\left(\left(\varphi_{1}, \varphi_{2}\right)\right) \wedge \varphi_{1}$ is consistent, then $\Delta_{\mu}\left(\left(\varphi_{1}, \varphi_{2}\right)\right) \wedge \varphi_{2}$ is consistent

(IC5) $\Delta_{\mu}\left(E_{1}\right) \wedge \Delta_{\mu}\left(E_{2}\right) \models \Delta_{\mu}\left(E_{1} \sqcup E_{2}\right)$

(IC6) If $\Delta_{\mu}\left(E_{1}\right) \wedge \Delta_{\mu}\left(E_{2}\right)$ is consistent, then $\Delta_{\mu}\left(E_{1} \sqcup E_{2}\right) \models \Delta_{\mu}\left(E_{1}\right) \wedge \Delta_{\mu}\left(E_{2}\right)$

(IC7) $\Delta_{\mu_{1}}(E) \wedge \mu_{2} \models \Delta_{\mu_{1} \wedge \mu_{2}}(E)$

(IC8) If $\Delta_{\mu_{1}}(E) \wedge \mu_{2}$ is consistent,

then $\Delta_{\mu_{1} \wedge \mu_{2}}(E) \models \Delta_{\mu_{1}}(E) \wedge \mu_{2}$

(Maj) $\exists n \geq 1 \Delta_{\mu}\left(E_{1} \sqcup E_{2}^{n}\right) \models \Delta_{\mu}\left(E_{2}\right)$

Definition 2. A function $E \mapsto \preceq_{E}$ that maps each profile $E$ to a total preorder over worlds $\preceq_{E}$ is called a syncretic assignment iff:

1. If $\omega, \omega^{\prime} \models \bigwedge E$, then $\omega \simeq_{E} \omega^{\prime}$

2. If $\omega=\bigwedge E$ and $\omega^{\prime} \mid \models \wedge E$, then $\omega \prec_{E} \omega^{\prime}$

3. If $E_{1} \equiv E_{2}$, then $\preceq_{E_{1}}=\preceq_{E_{2}}$

4. $\forall \omega \models \varphi_{1}, \exists \omega^{\prime} \models \varphi_{2}, \omega^{\prime} \preceq_{\varphi_{1} \sqcup \varphi_{2}} \omega$

5. If $\omega \preceq_{E_{1}} \omega^{\prime}$ and $\omega \preceq_{E_{2}} \omega^{\prime}$, then $\omega \preceq_{E_{1} \sqcup E_{2}} \omega^{\prime}$

6. If $\omega \preceq_{E_{1}} \omega^{\prime}$ and $\omega \prec_{E_{2}} \omega^{\prime}$, then $\omega \prec_{E_{1} \sqcup E_{2}} \omega^{\prime}$

A syncretic assignment is called a majority assignment iff:

7. If $\omega \prec_{E_{2}} \omega^{\prime}$, then $\exists n, \omega \prec E_{1} \sqcup E_{2}^{n} \omega^{\prime}$

Any IC merging operator can be represented by a syncretic assignment:

Theorem 1. (Konieczny and Pino Pérez 2002) A merging operator $\Delta$ is an IC merging operator (resp. majority merging operator) if and only if there exists a syncretic assignment (resp. majority assignment) that associates to every profile $E$ a total preorder $\preceq_{E}$ such that for any formula $\mu$,

$$
\left[\left[\Delta_{\mu}(E)\right]=\min (\llbracket \mu \rrbracket], \preceq_{E}\right)
$$

Observation 1. An analysis of the proof of this theorem reveals that:

- Postulates (IC0), (IC1), (IC7), (IC8) are enough to obtain the representation by an assignment $E \mapsto \preceq_{E}$ where $\preceq_{E}$ is a total preorder over interpretations.

- Modulo the postulates mentioned in the previous point (which allow the representation) we have: (IC2) is equivalent to properties ${ }^{2} 1$ and 2; (IC3) is equivalent to property 3 ; (IC4) is equivalent to property 4; (IC5) is equivalent to property 5; (IC6) is equivalent to property 6; (Maj) is equivalent to property 7 .

An interesting way to define concrete IC merging operators is to start from a distance $d$ between worlds and an aggregation function $f$ (see (Konieczny and Pino Pérez 2011)), in order to construct a syncretic assignment, and then to use the equation in Theorem 1 for defining the operator. More precisely:

\footnotetext{
${ }^{2}$ The properties are those of syncretic assignments (Definition 2).
}

Definition 3. Let $d: \Omega \times \Omega \rightarrow \mathbb{R}$ and $f: \bigcup_{n} \mathbb{R}^{n} \rightarrow \mathbb{R}$ be a distance $e^{3}$ between worlds and an aggregation function respectively. Suppose that $E=\left(\varphi_{1}, \ldots, \varphi_{n}\right)$ is a profile.

- $d\left(\omega, \varphi_{i}\right)=\min _{\omega^{\prime} \models \varphi_{i}} d\left(\omega, \omega^{\prime}\right)$

- $d^{f}(\omega, E)=f_{\varphi_{i} \in E}\left\{d\left(\omega, \varphi_{i}\right)\right\}$

- $\omega \preceq_{E}^{d, f} \omega^{\prime}$ iff $d^{f}(\omega, E) \leq d^{f}\left(\omega^{\prime}, E\right)$

Given a profile $E$ and a formula $\mu$, the distance-based merging operator $\Delta_{\mu}^{d, f}(E)$ is defined as $\llbracket\left[\Delta_{\mu}^{d, f}(E) \rrbracket=\right.$ $\min \left(\left[[\mu], \preceq_{E}^{d, f}\right)\right.$.

Usual distances are the drastic distance $\left(d_{D}\left(\omega, \omega^{\prime}\right)=0\right.$ if $\omega=\omega^{\prime}$ and 1 otherwise), and the Hamming distance $\left(d_{H}\left(\omega, \omega^{\prime}\right)=n\right.$ if $\omega$ and $\omega^{\prime}$ differ on $n$ variables). Usual aggregation functions are the sum, the leximax; the sum of the $\mathrm{n}^{\text {th }}$ powers, the leximin etc. All these aggregation functions satisfy the properties that allow to obtain IC merging operators (Konieczny, Lang, and Marquis 2004; Konieczny and Pino Pérez 2011). When the drastic distance $d_{D}$ is used, we refer to the IC merging operators $\Delta^{d_{D}, f}$ as the drastic merging operator (these operators are all equivalent to $\Delta^{d_{D}, \Sigma}$, see Corollary 1$)$.

\section{Borda Rules}

We have deliberately chosen the plural in the title of this section because in Social Choice Theory there are two ways for defining the Borda rule. They are equivalent when the preferences of the voters are linear orders. But they are not equivalent if the preferences of the voters are total preorders as we will see below.

We recall some of the basic notions on social choice theory before introducing the Borda rules. From now on, $N=\{1,2, \ldots, n\}$ will denote a finite set of voters and $X=\{x, y, z, \ldots\}$ will denote a finite set of alternatives. The ballot (or preference) of a voter $i$ will be given by a total preorder $\preceq_{i}$ over $X$. The meaning of $x \preceq_{i} y$ is that the agent $i$ prefers the alternative $x$ to the alternative $y .{ }^{4} \mathrm{~A}$ profile is an $n$-tuple $P=\left(\preceq_{1}, \preceq_{2}, \ldots, \preceq_{n}\right)$, which collects the ballots of all the voters in $N$, in an ordered manner. We will say that $P$ is a linear profile if each $\preceq_{i}$ in $P$ is a linear order over $X$. A welfare choice function is a map $W$ sending a profile $P$ into a total preorder $\preceq_{P}$, the social preference associated to profile $P$.

Let us now define the scoring Borda rule :

Definition 4. Given a profile $P=\left(\preceq_{1}, \ldots, \preceq_{n}\right)$ on a set of alternatives $X=\left\{x_{1}, \ldots, x_{m}\right\}$ and an alternative $a \in X$, the borda score of $a$ is the integer $r_{P}^{s}(a)=\sum_{i=1}^{n} r_{\unlhd_{i}}(a)$. Now define $\preceq_{P}^{s}$ by putting $a \preceq_{P}^{s} b$ iff $r_{P}^{s}(a) \leq r_{P}^{s}(b)$. Finally, the scoring Borda rule, $B^{s}$, is the welfare choice function $B^{s}(P)=\preceq_{P}^{s}$.

\footnotetext{
${ }^{3}$ Formally, only a pseudo-distance is required (triangle inequality is not necessary).

${ }^{4}$ We make this choice, converse to what is usually done in social choice, in order to be coherent with the orders used for belief revision.
} 
Example 1. Consider the set of alternatives $X=$ $\left\{x_{1}, x_{2}, x_{3}\right\}$ and the following profile $P=\left(\preceq_{1}, \preceq_{2}\right)$ where $x_{1} \prec_{1} x_{2} \simeq_{1} x_{3}$ and $x_{2} \prec_{2} x_{1} \prec_{2} x_{3}$. It is easy to see that $r_{P}^{s}\left(x_{1}\right)=1=r_{P}^{s}\left(x_{2}\right)$ and $r_{P}^{s}\left(x_{3}\right)=3$. Thus, $x_{1} \simeq_{P}^{s} x_{2} \prec_{P}^{s} x_{3}$.

Now we define the beta Borda rule (or comparison by pairs Borda rule).

Definition 5. Let $X$ be the set of alternatives $\left\{x_{1}, \ldots, x_{m}\right\}$. The score by comparison by pairs $(\beta)$ of the alternative $x_{i}$ in the profile $P=\left(\preceq_{1}, \ldots, \preceq_{n}\right)$ is defined as follows: $\beta_{i}(P)=\sum_{j=1}^{m}\left(\pi_{i j}(P)-\pi_{j i}(P)\right)$.

When $P$ is clear of the context we simply write $\beta_{i}$ instead of $\beta_{i}(P)$.

Now we put $x_{i} \preceq_{P}^{\beta} x_{j}$ iff $\beta_{i} \geq \beta_{j}$. Finally, the beta Borda rule $B^{\beta}$ is defined by putting $B^{\beta}(P)=\preceq_{P}^{\beta}$.

Example 2. Consider $X=\left\{x_{1}, x_{2}, x_{3}\right\}$ and $P$ as in Example 1. It is easy to see that $\beta_{1}(P)=2, \beta_{2}(P)=1$ and $\beta_{3}(P)=-3$, that is $x_{1} \prec_{P}^{\beta} x_{2} \prec_{P}^{\beta} x_{3}$.

We can now state that the two definitions are equivalent for linear orders, but not for general preorders. This result is known in social choice, and is mentionned in (Young 1974) but without proof. So we put it here for completeness.

Proposition 1. For every linear profile $P$ we have $B^{s}(P)=$ $B^{\beta}(P)$. This equality is not true in general, i.e. there exists a profile $P$ such that $B^{s}(P) \neq B^{\beta}(P)$.

Proof. We observe that an alternative way to calculate $\sum_{j=1}^{m} \pi_{i j}(P)$ is, for each $k \in\{1, \ldots, n\}$, to count the number of alternatives $y$ s.t. $x_{i} \prec_{k} y$ and then make the sum of these numbers over $k \in\{1, \ldots, n\}$. Analogously, an alternative way to calculate $\sum_{j=1}^{m} \pi_{j i}(P)$ is, for each $k \in\{1, \ldots, n\}$, count the number of alternatives $y$ s.t. $y \prec_{k} \quad x_{i}$ and then make the sum of these numbers over $k \in\{1, \ldots, n\}$. Note that if $\preceq_{k}$ is a linear order, the number of alternatives $y$ s.t. $x_{i} \prec_{k} y$ is exactly $m-1-r_{\preceq_{k}}\left(x_{i}\right)$ and the number of alternatives $y$ s.t. $y \prec_{k} \quad x_{i}$ is exactly $r_{\preceq}\left(x_{i}\right)-1$. Thus,

$\sum_{j=1}^{n_{m}} \pi_{i j}(P)=\sum_{k=1}^{n} m-1-r_{\preceq_{k}}\left(x_{i}\right)=n m-n-r_{P}^{s}\left(x_{i}\right)$, $\sum_{j=1}^{m} \pi_{j i}(P)=\sum_{k=1}^{n} r_{\preceq_{k}}\left(x_{i}\right)-1=r_{P}^{s}\left(x_{i}\right)-n$ and $\beta_{i}=\sum_{j=1}^{m} \pi_{i j}(P)-\sum_{j=1}^{m} \pi_{j i}(P)=n m-2 r_{P}^{s}\left(x_{i}\right)$. Therefore, $x_{i} \preceq_{P}^{\beta} x_{j}$ iff $\beta_{i} \geq \beta_{j}$ iff $n m-2 r_{P}^{s}\left(x_{i}\right) \geq$ $n m-2 r_{P}^{s}\left(x_{j}\right)$ iff $r_{P}^{s}\left(x_{i}\right) \leq r_{P}^{s}\left(x_{j}\right)$ iff $x_{i} \preceq_{P}^{s} x_{j}$.

The computations of Examples 1 and 2 show that $B^{s}(P) \neq B^{\beta}(P)$.

An interesting result by Young (1974) is an axiomatic characterization of Borda rule for linear profiles. Let us introduce four axioms for a social welfare function $W$ :

Neutrality: For every permutation $\sigma$ over the alternatives, $W(\sigma(P))=\sigma(W(P))$.

Faithfulness: For every singleton profile $(\preceq), W((\preceq))=\preceq$. Consistency: Assume $P_{1}$ and $P_{2}$ are profiles over disjoint voters. If $\min \left(W\left(P_{1}\right)\right) \cap \min \left(W\left(P_{2}\right)\right) \neq \emptyset$, then $\min \left(W\left(P_{1} \sqcup P_{2}\right)\right)=\min \left(W\left(P_{1}\right)\right) \cap \min \left(W\left(P_{2}\right)\right)$.
Cancellation: If for every pair of alternatives $x_{i}, x_{j}$ we have $\pi_{i j}(P)=\pi_{j i}(P)$ then $W(P)$ is the flat total preorder ${ }^{5}$.

Theorem 2. (Young 1974) Restrained to linear profiles, the only social welfare function satisfying neutrality, faithfulness, consistency and cancellation is the Borda rule $B^{s}$.

So on linear profiles the two definitions of Borda rules coincide, and this rule is characterized exactly by those four axioms.

\section{Borda-like Merging}

In this section we will use the two definitions of the previous section (which we know, from Proposition 1, differ in the general case) in order to define merging operators. We just need a generating function that associates a total preorder on interpretations to any base $\varphi$ :

Definition 6. We call generating function any function $\triangleleft$ that associates to each belief base $\varphi$ a total preorder on interpretations $\preceq_{\varphi}$ such that $\min (\Omega, \preceq \varphi)=[[\varphi]]$ and $\preceq_{\varphi}=\preceq_{\varphi^{\prime}}$ whenever $\varphi \equiv \varphi^{\prime}$.

Note that $\triangleleft$ can be provided by a revision operator $\circ$ or a merging operator $\Delta$ since, from the representation theorems in terms of faifthful/syncretic assignments, they allow to associate a preorder to each belief base $\varphi$. Note that a distance $d$ between interpretations is also a manner to define a preorder for each belief base $\varphi$, with $\omega \preceq_{\varphi} \omega^{\prime}$ if and only if $d(\omega, \varphi) \leq d\left(\omega^{\prime}, \varphi\right)$.

Once a generating function $\triangleleft$ is considered, it is easy to define a profile of preorders over $\Omega$ (the elements of $\Omega$ are viewed as alternatives) $P_{E}=\left(\preceq_{\varphi_{1}}, \ldots, \preceq_{\varphi_{n}}\right)$ from a profile of belief bases $E=\left(\varphi_{1}, \ldots, \varphi_{n}\right)$.

Definition 7. Given a generating function $\triangleleft$ and a profile $P_{E}^{\triangleleft}=\left(\preceq_{\varphi_{1}}, \ldots, \preceq_{\varphi_{n}}\right)$ over $\Omega$ associated to $E=$ $\left(\varphi_{1}, \ldots, \varphi_{n}\right)$ with $\triangleleft$, we consider the assignment $P_{E}^{\triangleleft} \mapsto \preceq_{P_{E}}^{\beta}$ (see Definition 5). The $\beta$-Borda merging operator $\Delta^{\beta_{\triangleleft}}$ is defined by:

$$
\left[\left[\Delta_{\mu}^{\beta_{\triangleleft}}(E)\right]=\min \left([[\mu]], \preceq_{P_{E}}^{\beta}\right)\right.
$$

We say that a merging operator is based on pairwise comparison if it is identical to its $\beta$-Borda merging version :

Definition 8. Let $\Delta$ be a merging operator and $\triangleleft_{\Delta}$ the generating function associated to $\Delta$. We say that $\Delta$ is based on pairwise comparisons iff $\Delta=\Delta^{\beta_{\triangleleft}}$.

In an analogous way, using the scoring Borda welfare function $B^{s}$ of Definition 4, we can define another merging operator, which transforms each preorder into a score function for each base, and the score of an interpretation is the sum of all its scores. This way to proceed can be related to the usual $\Delta^{d, \Sigma}$ operators, but instead of using a distance, we use a more qualitative information provided by the preorder: Definition 9. Given a generating function $\triangleleft$, a profile $P_{E}=$ $\left(\preceq \varphi_{1}, \ldots, \preceq \varphi_{n}\right)$ over $\Omega$ associated to $E=\left(\varphi_{1}, \ldots, \varphi_{n}\right)$ with $\triangleleft$, we consider the assignment $E \mapsto \preceq_{P_{E}}^{s}$ (see Definition 4). The $s$-Borda merging operator $\Delta^{s \triangleleft}$ is defined by:

$$
\left[\left[\Delta_{\mu}^{s_{\triangleleft}}(E)\right]\right]=\min \left([[\mu]], \preceq_{P_{E}}^{s}\right)
$$

${ }^{5}$ The flat total preorder over $X$, is the unique total preorder $\preceq$ such that for all $x, y$ in $X$, we have $x \preceq y$. 


\section{Example}

In this section we provide an example for illustrating the difference of behaviour between $\beta$-Borda operators, $s$-Borda operators and classical distance-based merging operators.

Consider the profile $E=\left(\varphi_{1}, \varphi_{2}, \varphi_{3}\right)$, with $\left[\varphi_{1} \rrbracket=\right.$ $\{111,001\},\left[\varphi_{2} \rrbracket=\{011,110\}\right.$, and $\left.\left[\varphi_{3}\right]\right]=\{000\}$. We do not consider any integrity constraint, so $\mu=\top$.

We will consider the distance-based merging operator $\Delta^{d_{H}^{\rho}, \Sigma}$, that is, the operator for which the sum is the aggregation function, and the distance is the weighted Hamming distance $d_{H}^{\rho}$, defined below:

Definition 10. Let $\rho: \mathcal{P} \rightarrow \mathbb{R}^{*}$ be a function that associates to any propositional variable in the language a nonnull weight. Then the weighted-Hamming distance $d_{H}^{\rho}$ between two interpretations $\omega$ and $\omega^{\prime}$ is defined as:

$$
d_{H}^{\rho}\left(\omega, \omega^{\prime}\right)=\sum_{x \in \mathcal{P}, \omega(x) \neq \omega^{\prime}(x)} \rho(x)
$$

Using such a distance is very natural when all propositional variables do not have the same importance. Such is the case if the base can be split in several topics (encoded by subsets of propositional variables), with some topics being more important than others.

Note that the standard Hamming distance is obtained in the particular case where all weights are equal to 1 .

In this example we will use the weight function $\rho=$ $(1,3,6)$. And we will look at the results of the merging of the profile $E$ for the operator $\Delta^{d_{H}^{\rho}, \Sigma}$, for its $s$-Borda and $\beta$ Borda versions where the generating function $\varangle$ is given by $\Delta d_{H}^{\rho}, \Sigma$.

Tables 1, 2, 3 sum up the respective computations.

Then one can check that the result for the distance-based operator is $\left[\left[\Delta^{d_{H}^{\rho}, \Sigma}(E)\right]=\{001\}\right.$. Whereas for its $s$-Borda version the exact values of the distance are not used, just the plausibility order from each base is considered, that leads us to a different result: $\left[\left[\Delta^{s} \triangleleft(E)\right]\right]=\{000,110\}$. Finally, using the $\beta$-Borda version of this operator, we compute the relative positions of all couples of interpretations via the $\beta_{i}$, and we obtain as result: $\left.\left[\Delta^{\beta} \triangleleft(E)\right]\right]=\{110\}$.

Finally, let us note that there is not, in general, a logical correlation between the operators $\Delta^{d_{H}^{\rho}, \Sigma}, \Delta^{s}$ and $\Delta^{\beta} \hookrightarrow$. In this example we can already see that this is the case between $\Delta^{d_{H}^{\rho}, \Sigma}$ and the two other operators, but we have $\Delta^{\beta} \triangleleft(E) \vdash$ $\Delta^{s} \triangleleft(E)$. To see that it is not always the case let us do the merging under the constraints $[\mu] \rrbracket=\{010,111\}$. From tables 2 and 3 we can easily see that $\left[\left[\Delta_{\mu}^{s}(E)\right]=\{010\}\right.$ and $\left[\left[\Delta_{\mu}^{\beta}(E)\right]=\{111\}\right.$ Thus, in general, it is not the case that $\Delta_{\mu}^{s} \triangleleft(E) \vdash \Delta_{\mu}^{\beta} \triangleleft(E)$ nor $\Delta_{\mu}^{\beta} \triangleleft(E) \vdash \Delta_{\mu}^{s} \triangleleft(E)$.

\section{Properties of Borda-like Merging}

We will now study the logical properties of the two abovedefined families of operators.

First we can show that $s$-Borda merging operators satisfy all IC merging postulates except (IC4). This fact is a direct consequence of a result of (Konieczny and Pino Pérez 2002):

Proposition 2. A merging operator $\Delta^{s_{\triangleleft}}$ satisfies (ICO-IC3) and (IC5-IC8).

\begin{tabular}{rcccr} 
& $\varphi_{1}$ & $\varphi_{2}$ & $\varphi_{3}$ & dist $_{\rho, \Sigma}$ \\
\hline 000 & 6 & 4 & 0 & 10 \\
001 & 0 & 3 & 6 & 9 \\
010 & 7 & 1 & 3 & 11 \\
011 & 1 & 0 & 9 & 10 \\
100 & 7 & 3 & 1 & 11 \\
101 & 1 & 4 & 7 & 12 \\
110 & 6 & 0 & 4 & 10 \\
111 & 0 & 1 & 10 & 11
\end{tabular}

Table 1: $\Delta^{d_{H}^{\rho}, \Sigma}(E)$

\begin{tabular}{ccccr} 
& $\varphi_{1}$ & $\varphi_{2}$ & $\varphi_{3}$ & dist $_{\rho, s}$ \\
\hline 000 & 2 & 3 & 0 & 5 \\
001 & 0 & 2 & 4 & 6 \\
010 & 3 & 1 & 2 & 6 \\
011 & 1 & 0 & 6 & 7 \\
100 & 3 & 2 & 1 & 6 \\
101 & 1 & 3 & 5 & 9 \\
110 & 2 & 0 & 3 & 5 \\
111 & 0 & 1 & 7 & 8 \\
& \multicolumn{5}{c}{ Table 2: $\Delta^{s} \triangleleft(E)$} &
\end{tabular}

So we obtain all the usual rationality postulates except (IC4) which requires some symmetry between the bases, that we can not ensure here.

Now note that we obtain the same result for $\beta$-Borda operators:

Proposition 3. A merging operator $\Delta^{\beta_{\triangleleft}}$ satisfies (ICO-IC3) and (IC5-IC8).

Proof. Given that the operator $\Delta^{\beta_{\triangleleft}}$ is defined by the assignment $E \mapsto \preceq_{P_{E}}^{\beta}$ (cf. Definition 7), Observation 1 tells us that (IC0), (IC1), (IC7) and (IC8) are satisfied. Moreover, by Observation 1, in order to prove (IC2), we have to prove that the assignment satisfies properties 1 and 2; in order to prove (IC3) we have to prove that the assignment satisfies property 3 ; in order to prove (IC5) we have to prove that the assignment satisfies property 5 and, finally, in order to prove (IC6) we have to prove that the assignment satisfies property 6 . Then, we proceed to prove properties $1-3,5$ and 6 .

\begin{tabular}{cccc} 
& $\pi_{i j}$ & $\pi_{j i}$ & $\beta_{i}$ \\
\hline 000 & 9 & 10 & -1 \\
001 & 11 & 8 & 3 \\
010 & 9 & 10 & -1 \\
011 & 11 & 8 & 3 \\
100 & 8 & 11 & -3 \\
101 & 6 & 13 & -7 \\
110 & 12 & 7 & 5 \\
111 & 10 & 9 & 1
\end{tabular}

Table 3: $\Delta^{\beta} \hookrightarrow(E)$ 
Property 1: We want to show that if $\omega_{1} \models \bigwedge E$ and $\omega_{2} \models$ $\bigwedge E$, then $\omega_{1} \simeq_{P_{E}}^{\beta} \omega_{2}$ where $P_{E}=\left(\preceq_{\varphi_{1}}, \ldots, \preceq \varphi_{n}\right)$. Thus, we have to show that $\beta_{1}\left(P_{E}\right)=\beta_{2}\left(P_{E}\right)$. Let's compute $\beta_{1}\left(P_{E}\right)$ and $\beta_{2}\left(P_{E}\right)$ :

$$
\beta_{1}\left(P_{E}\right)=\sum_{1 \leq j \leq m}\left(\pi_{1 j}\left(P_{E}\right)-\pi_{j 1}\left(P_{E}\right)\right)
$$

and

$$
\beta_{2}\left(P_{E}\right)=\sum_{1 \leq j \leq m}\left(\pi_{2 j}\left(P_{E}\right)-\pi_{j 2}\left(P_{E}\right)\right)
$$

As $\forall i, \pi_{i i}\left(P_{E}\right)=0$, we can easily deduce that: $\beta_{1}\left(P_{E}\right)=$ $\left(\pi_{12}\left(P_{E}\right)-\pi_{21}\left(P_{E}\right)\right)+\sum_{3 \leq j \leq m}\left(\pi_{1 j}\left(P_{E}\right)-\pi_{j 1}\left(P_{E}\right)\right)$ and $\beta_{2}\left(P_{E}\right)=\left(\pi_{21}\left(P_{E}\right)-\pi_{12}\left(P_{E}\right)\right)+\sum_{3 \leq j \leq m}\left(\pi_{2 j}\left(P_{E}\right)-\right.$ $\left.\pi_{j 2}\left(P_{E}\right)\right)$. We have $\omega_{1}=\bigwedge E$, then, $\forall \varphi_{i} \in E, \omega_{1}=\varphi_{i}$, thus $\forall \omega_{j} \in \Omega, \omega_{1} \preceq_{\varphi_{i}} \omega_{j}$ (no interpretation $\omega_{j} \in \Omega$ can be better than $\left.\omega_{1}\right)$, then $\pi_{j 1}\left(P_{E}\right)=0 \quad$ (1).

The same reasoning holds for $\omega_{2}$, and $\forall j \pi_{j 2}\left(P_{E}\right)=0 \quad$ (2). From (1) and (2) we deduce that:

$$
\beta_{1}\left(P_{E}\right)=\sum_{3 \leq j \leq m} \pi_{1 j}\left(P_{E}\right) \text { and } \beta_{2}\left(P_{E}\right)=\sum_{3 \leq j \leq m} \pi_{2 j}\left(P_{E}\right)
$$

We have $\forall \varphi_{i} \in E, \omega_{1}=\varphi_{i}$ and $\omega_{2}=\varphi_{i}$. So, $\forall \omega_{j} \in$ $\Omega, \omega_{1} \prec_{\varphi_{i}} \omega_{j}$ if and only if $\omega_{2} \prec_{\varphi_{i}} \omega_{j}$. From this, we can deduce that $\pi_{1 j}\left(P_{E}\right)=\pi_{2 j}\left(P_{E}\right)$, and $\sum_{3 \leq j \leq m} \pi_{1 j}\left(P_{E}\right)=$ $\sum_{3 \leq j \leq m} \pi_{2 j}\left(P_{E}\right)$. This gives us $\beta_{1}\left(P_{E}\right)=\beta_{2}\left(P_{E}\right)$, thus $\omega_{1} \simeq_{P_{E}}^{\beta} \omega_{2}$.

Property 2: We have to show that if $\omega_{1} \models \wedge E$ and $\omega_{2} \not \models$ $\bigwedge E$, then $\omega_{1} \prec_{P_{E}}^{\beta} \omega_{2}$. Thus, we have to prove $\beta_{1}\left(P_{E}\right)>$ $\beta_{2}\left(P_{E}\right)$, that is,

$$
\sum_{1 \leq j \leq m} \pi_{1 j}\left(P_{E}\right)-\pi_{j 1}\left(P_{E}\right)>\sum_{1 \leq j \leq m} \pi_{2 j}\left(P_{E}\right)-\pi_{j 2}\left(P_{E}\right)
$$

Since $\omega_{1} \models \bigwedge E, \forall \varphi_{i} \in E, \forall \omega_{j} \in \Omega, \omega_{1} \preceq_{\varphi_{i}} \omega_{j}$, thereby $\pi_{j 1}\left(P_{E}\right)=0$, thus Equation 1 is equivalent to:

$$
\sum_{1 \leq j \leq m} \pi_{1 j}\left(P_{E}\right)>\sum_{1 \leq j \leq m} \pi_{2 j}\left(P_{E}\right)-\pi_{j 2}\left(P_{E}\right)
$$

or to:

$$
\begin{gathered}
\pi_{12}\left(P_{E}\right)+\sum_{3 \leq j \leq m} \pi_{1 j}\left(P_{E}\right)> \\
\left(\pi_{21}\left(P_{E}\right)-\pi_{12}\left(P_{E}\right)\right)+\sum_{3 \leq j \leq m} \pi_{2 j}\left(P_{E}\right)-\pi_{j 2}\left(P_{E}\right)
\end{gathered}
$$

To show the inequality below it is sufficient to show:

$$
\pi_{12}\left(P_{E}\right)>\left(\pi_{21}\left(P_{E}\right)-\pi_{12}\left(P_{E}\right)\right)
$$

and

$$
\sum_{3 \leq j \leq m} \pi_{1 j}\left(P_{E}\right) \geq \sum_{3 \leq j \leq m} \pi_{2 j}\left(P_{E}\right)-\pi_{j 2}\left(P_{E}\right)
$$

For (2) we already know that $\pi_{21}\left(P_{E}\right)=0$. We know also that $\forall \varphi_{i} \in E, \omega_{1} \preceq_{\varphi_{i}} \omega_{2}$ and $\omega_{2} \not \models E$, then $\exists \varphi_{i} \in E$, s.t. $\omega_{2} \not \models \varphi_{i}$, thus $\omega_{1} \prec_{\varphi_{i}} \omega_{2}$, which implies that $\pi_{12}\left(P_{E}\right)>0$, then $\pi_{12}\left(P_{E}\right)>-\pi_{12}\left(P_{E}\right)$ and (2) is shown.

For (3), $\forall \varphi_{i} \in E, \omega_{1} \models \varphi_{i}$, then $\omega_{1} \preceq_{\varphi_{i}} \omega_{2}$, and $\left(\omega_{2} \preceq_{\varphi_{i}}\right.$ $\left.\omega_{j}\right) \Rightarrow\left(\omega_{1} \preceq_{\varphi_{i}} \omega_{j}\right)$ (the bases that prefer $\omega_{2}$ over $\omega_{j}$ should also prefer $\omega_{1}$ over $\left.\omega_{j}\right)$, thus

$$
\pi_{1 j}\left(P_{E}\right) \geq \pi_{2 j}\left(P_{E}\right)
$$

and also

$$
\sum_{3 \leq j \leq m} \pi_{1 j}\left(P_{E}\right) \geq \sum_{3 \leq j \leq m} \pi_{2 j}\left(P_{E}\right)
$$

We know that $\pi_{j 2}\left(P_{E}\right) \geq 0$, then $\sum_{3 \leq j \leq m} \pi_{j 2}\left(P_{E}\right) \geq 0$, thus

$$
\sum_{3 \leq j \leq m} \pi_{1 j}\left(P_{E}\right) \geq \sum_{3 \leq j \leq m} \pi_{2 j}\left(P_{E}\right)-\pi_{j 2}\left(P_{E}\right)
$$

which shows that (3) is true. As a consequence, $\omega_{1} \prec_{P_{E}}^{\beta} \omega_{2}$. Property 3: We want to show that if $E_{1} \equiv E_{2}$, then $\preceq_{P_{E_{1}}}^{\beta}=\preceq_{P_{E_{2}}}^{\beta}$. Suppose that $E_{1}=\left(\varphi_{1}, \ldots, \varphi_{n}\right)$ and $E_{2}=\left(\varphi_{1}^{\prime}, \ldots, \varphi_{n}^{\prime}\right)$ and for every $i \in\{1, \ldots, n\}, \varphi_{i} \equiv$ $\varphi_{i}^{\prime}$. Since $\triangleleft$ is a generating function, for every $i \in$ $\{1, \ldots, n\}, \preceq_{\varphi_{i}}=\preceq_{\varphi_{i}^{\prime}}$. Therefore, $P_{E_{1}}=P_{E_{2}}$ and trivially $\preceq_{P_{E_{1}}}^{\beta}=\preceq_{P_{E_{2}}}^{\beta}$.

Before proceeding to the proof of conditions 5 and 6 , we observe the following fact. If $E_{1}$ and $E_{2}$ are two profiles, we have for every $k \in\{1, \ldots, m\}$

$$
\beta_{k}\left(P_{E_{1}} \sqcup E_{2}\right)=\beta_{k}\left(P_{E_{1}}\right)+\beta_{k}\left(P_{E_{2}}\right)
$$

Property 5: We want to show that if $\omega_{1} \preceq_{P_{E_{1}}}^{\beta} \omega_{2}$ and $\omega_{1} \preceq_{P_{E_{2}}}^{\beta} \omega_{2}$, then $\omega_{1} \preceq_{P_{E_{1}} \sqcup E_{2}}^{\beta} \omega_{2}$. By assumption we have $\omega_{1} \preceq_{E_{1}}^{\beta} \omega_{2}$, i.e. $\beta_{1}\left(P_{E_{1}}\right) \geq \beta_{2}\left(P_{E_{1}}\right)(*)$. We have also $\omega_{1} \preceq_{E_{2}}^{\beta} \omega_{2}$, i.e. $\beta_{1}\left(P_{E_{2}}\right) \geq \beta_{2}\left(P_{E_{2}}\right)(* *)$. From $(*)$ and (**) we have, $\beta_{1}\left(P_{E_{1}}\right)+\beta_{1}\left(P_{E_{2}}\right) \geq \beta_{2}\left(P_{E_{1}}\right)+\beta_{2}\left(P_{E_{2}}\right)$. From this and Equation 2 we obtain, $\beta_{1}\left(P_{E_{1}} \sqcup E_{2}\right) \geq$ $\beta_{2}\left(P_{E_{1} \sqcup E_{2}}\right)$. Thus, $\omega_{1} \preceq_{P_{E_{1}} \sqcup E_{2}}^{\beta} \omega_{2}$.

Property 6: We want to show that if $\omega_{1} \preceq_{P_{E_{1}}}^{\beta} \omega_{2}$ and $\omega_{1} \prec_{P_{E_{2}}}^{\beta} \omega_{2}$, then $\omega_{1} \prec_{P_{E_{1}} \sqcup E_{2}}^{\beta} \omega_{2}$. As in the previous point, from the assumptions, we obtain $\beta_{1}\left(P_{E_{1}}\right) \geq \beta_{2}\left(P_{E_{1}}\right)$ (1) and $\beta_{1}\left(P_{E_{2}}\right)>\beta_{2}\left(P_{E_{2}}\right)$ (2). From (1) and (2) we have, $\beta_{1}\left(P_{E_{1}}\right)+\beta_{1}\left(P_{E_{2}}\right)>\beta_{2}\left(P_{E_{1}}\right)+\beta_{2}\left(P_{E_{2}}\right)$. From this and Equation 2 we obtain, $\beta_{1}\left(P_{E_{1} \sqcup E_{2}}\right)>\beta_{2}\left(P_{E_{1} \sqcup E_{2}}\right)$. Thus, $\omega_{1} \prec_{P_{E_{1} \sqcup E_{2}}}^{\beta} \omega_{2}$.

This definition of $\beta$-Borda (pairwise comparison) operators allows us to provide a characterization of the drastic merging operator.

Theorem 3. Let $\Delta^{d, f}$ be a distance-based operator. $\Delta^{d, f}$ is based on pairwise comparisons if and only if it is the drastic operator.

The proof is organized in three lemmas.

Lemma 1. If a distance $d$ is not the drastic distance then there exist $x, y$ and $z$ such that $d(x, y)<d(x, z)$. 
Proof. By assumption there exist $\omega_{1}, \omega_{2}, \omega_{3}$ and $\omega_{4}$ such that $0<d\left(\omega_{1}, \omega_{2}\right)<d\left(\omega_{3}, \omega_{4}\right)$. Put $a=d\left(\omega_{1}, \omega_{2}\right)$. We consider $c=d\left(\omega_{1}, \omega_{3}\right)$. If $c=0$, then $\omega_{1}=\omega_{3}$ and defining $x=\omega_{1}, y=\omega_{2}$ and $z=\omega_{4}$, we have $d(x, y)<d(x, z)$. If $c \neq 0$, we consider two cases: $c=a$ or $c \neq a$. In the first case, $c=a$, we put $x=\omega_{3}, y=\omega_{1}$ and $z=\omega_{4}$ and we have $d(x, y)<d(x, z)$. In the second case, $c \neq a$, we can assume (w.l.o.g.) that $c<a$, then putting $x=\omega_{1}, y=\omega_{3}$ and $z=\omega_{2}$, we have $d(x, y)<d(x, z)$.

Lemma 2. If a distance $d$ is not the drastic distance $d_{D}$, then $\Delta^{d, f}$ is not based on pairwise comparisons.

Proof. If $d$ is not a drastic distance, necessarily the set of interpretations $\Omega=\left\{\omega_{1}, \ldots, \omega_{m}\right\}$ is such that $m \geq 4$. Moreover, by Lemma 1 , there are $\omega_{i}, \omega_{j}$, and $\omega_{k}$ such that $d\left(\omega_{i}, \omega_{j}\right)<d\left(\omega_{i}, \omega_{k}\right)$. Consider $\varphi_{\omega_{i}}$, the base such that $\left[\varphi_{\omega_{i}}\right]=\left\{\omega_{i}\right\}$. Then we have $0=d\left(\omega_{i}, \varphi_{\omega_{i}}\right)<$ $d\left(\omega_{j}, \varphi_{\omega_{i}}\right)<d\left(\omega_{k}, \varphi_{\omega_{i}}\right)$. Consider $\varphi_{\neg \omega_{i}}$ the base defined by $\varphi_{\neg \omega_{i}}=\neg \varphi_{\omega_{i}}$, in particular, $\left[\left[\varphi_{\neg \omega_{i}}\right]=\Omega \backslash\left\{\omega_{i}\right\}\right.$. Then $\forall \omega \neq \omega_{i}, d\left(\omega, \varphi_{\neg \omega_{i}}\right)=0$, whereas $d\left(\omega_{i}, \varphi_{\neg \omega_{i}}\right)>0$. Let $\omega_{h} \in \llbracket\left[\varphi_{\neg \omega_{i}} \rrbracket\right.$ s.t. $d\left(\omega_{i}, \omega_{h}\right)=\min _{\left.\omega \in \llbracket \varphi_{\neg \omega_{i}}\right]} d\left(\omega_{i}, \omega\right)=$ $d\left(\omega_{i}, \varphi_{\neg \omega_{i}}\right)$.

Observe that necessarily $d\left(\omega_{i}, \omega_{h}\right) \leq d\left(\omega_{i}, \omega_{j}\right)$. Therefore, $d\left(\omega_{i}, \omega_{h}\right) \leq d\left(\omega_{i}, \omega_{k}\right)$.

We have $d\left(\omega_{i}, \varphi_{\omega_{i}}\right)<d\left(\omega_{h}, \varphi_{\omega_{i}}\right)<d\left(\omega_{k}, \varphi_{\omega_{i}}\right)$ and $d\left(\omega_{h}, \varphi_{\neg \omega_{i}}\right)=d\left(\omega_{k}, \varphi_{\neg \omega_{i}}\right)<d\left(\omega_{i}, \varphi_{\neg \omega_{i}}\right)$.

Consider $E=\left(\varphi_{\omega_{i}}, \varphi_{\neg \omega_{i}}\right)$. Let us compute $\beta_{i}$ and $\beta_{h}$. Remember that $\beta_{i}$ counts the number of interpretations that are ranked worse than $\omega_{i}$ in all bases, minus the number of interpretations that are ranked better than $\omega_{i}$ in all bases. Here we have 2 bases $\varphi_{\omega_{i}}$ and $\varphi_{\neg \omega_{i}}$, so for $\varphi_{\omega_{i}}, \omega_{i}$ is better than all other interpretations, and it is the contrary for $\varphi_{\neg \omega_{i}}$. So $\beta_{i}=\Sigma_{p=1, p \neq i}^{p=m} \pi_{i p}-\Sigma_{p=1, p \neq i}^{p=m} \pi_{p i}$ $=(m-1)-(m-1)=0$.

Now we are going to calculate $\beta_{h}=\sum_{p=1, p \neq h}^{p=m} \pi_{h p}-$ $\sum_{p=1, p \neq h}^{p=m} \pi_{p h}$.

Let $l \in\{1, \ldots, m\}, l \neq i, l \neq k$ and $l \neq h$. As $\omega_{h} \equiv_{\varphi_{\neg \omega_{i}}} \omega_{l}, \pi_{h l}-\pi_{l h}=a_{h, l}$, with $a_{h, l}=0$ if $d\left(\omega_{h}, \varphi_{\omega_{i}}\right)=d\left(\omega_{l}, \varphi_{\omega_{i}}\right)$ or $a_{h, l}=1$ if $d\left(\omega_{h}, \varphi_{\omega_{i}}\right)<$ $d\left(\omega_{l}, \varphi_{\omega_{i}}\right)$. The case $a_{h, l}=-1$ is not possible because it corresponds to the situation where $d\left(\omega_{h}, \varphi_{\omega_{i}}\right)>d\left(\omega_{l}, \varphi_{\omega_{i}}\right)$, i.e. $d\left(\omega_{h}, \omega_{i}\right)>d\left(\omega_{l}, \omega_{i}\right)$, impossible by assumption $\left(d\left(\omega_{i}, \omega_{h}\right)=\min _{\omega \in \Omega} d\left(\omega_{i}, \omega\right)\right)$. So $\pi_{h l}-\pi_{l h} \geq 0$.

Furthermore, $\pi_{h i}-\pi_{i h}=0$ (since $\omega_{i} \models \varphi_{\omega_{i}}$ and $\omega_{h} \not \models \varphi_{\omega_{i}}$ ) and $\pi_{h k}-\pi_{k h}=1$ (since by hypothesis $d\left(\omega_{h}, \varphi_{\omega_{i}}\right)<$ $\left.d\left(\omega_{k}, \varphi_{\omega_{i}}\right)\right)$. So, $\beta_{h}>0$. Therefore, $\beta_{h}>\beta_{i}$, i.e. $\omega_{h} \prec_{E}^{\beta} \omega_{i}$.

On the other hand, by hypothesis $d\left(\omega_{i}, \varphi_{\neg \omega_{i}}\right)=d\left(\omega_{i}, \omega_{h}\right)$ and as $\omega_{i}$ is the only model of $\varphi_{\omega_{i}}$, then $d\left(\omega_{h}, \varphi_{\omega_{i}}\right)=$ $d\left(\omega_{i}, \omega_{h}\right)$. Therefore $\left(d\left(\omega_{i}, \varphi_{\omega_{i}}\right), \quad d\left(\omega_{i}, \varphi_{\neg \omega_{i}}\right)\right)=$ $\left(d\left(\omega_{h}, \varphi_{\neg \omega_{i}}\right), d\left(\omega_{h}, \varphi_{\omega_{i}}\right)\right)$. Thus, for any anonymous aggregation function $f$ used to compute the distance of $\omega_{i}$ and $\omega_{h}$ to $E$, we have the same output, i.e. $\omega_{i} \simeq_{\Delta^{d, f}} \omega_{h}$.

This proves that the preorders generated by the $\beta$-Borda operator and by the $\Delta^{d, f}$ operators may be different, and then the result holds.
Lemma 3. The drastic operator $\Delta^{d_{D}, f}$ is based on pairwise comparisons.

Proof. Consider a profile $E=\left(\varphi_{1}, \ldots \varphi_{n}\right)$ of $n$ bases and a set of $m$ interpretations $\left\{\omega_{1}, \ldots, \omega_{m}\right\}$. We denote by $E_{\preceq^{d_{D}}}=\left(\preceq_{1}^{d_{D}}, \ldots \preceq_{n}^{d_{D}}\right)$ the profile of preorders obtained from $E$ with the drastic distance $d_{D}$.

$\forall j, k \in\{1, \ldots, m\}, \pi_{j k}\left(E_{\prec^{d} D}\right)$ represents the number of preorders in $E_{\prec^{d_{D}}}$ which (strictly) prefer $\omega_{j}$ to $\omega_{k}$. As the drastic distance $d_{D}$ is used, we know that $\omega_{j} \prec_{\varphi} \omega_{k}$ if and only if $d_{D}\left(\omega_{j}, \varphi\right)<d_{D}\left(\omega_{k}, \varphi\right)$ i.e. if and only if $\omega_{j} \models \varphi$ and $\omega_{k} \not \models \varphi$. As a consequence:

$\pi_{k j}\left(E_{\prec^{d_{D}}}\right)=\left|\left\{i \in\{1, \ldots n\}: \omega_{k}=\varphi \wedge \omega_{j} \not \models \varphi\right\}\right|$.

$\beta_{k}\left(E_{\preceq^{d} D}\right)=\sum_{j=1, j \neq k}^{m} \pi_{k j}\left(E_{\preceq^{d} D}\right)-\pi_{j k}\left(E_{\preceq^{d} D}\right)$

$=\bar{\Sigma}_{j=1, j \neq k}^{m}\left|\left\{i \in\{1, \ldots n\}: \omega_{k} \models \varphi_{i} \wedge \omega_{j} \mid \models \varphi_{i}\right\}\right|-$ $\left|\left\{i \in\{1, \ldots n\}: \omega_{j} \models \varphi_{i} \wedge \omega_{k} \not \models \varphi_{i}\right\}\right|$

$=\Sigma_{j=1, j \neq k}^{m}\left|\left\{i \in\{1, \ldots n\}: \omega_{k} \models \varphi_{i} \wedge \omega_{j} \not \models \varphi_{i}\right\}\right|-$ $\Sigma_{j=1, j \neq k}^{m}\left|\left\{i \in\{1, \ldots n\}: \omega_{j} \models \varphi_{i} \wedge \omega_{k} \not \models \varphi_{i}\right\}\right|$

$\stackrel{=}{=} \sum_{i=1, \omega_{k} \models \varphi_{i}}^{n} \Sigma_{j=1, j \neq k, \omega_{j} \not \varphi_{i}}^{m} 1-$

$=\sum_{i=1, \omega_{k} \models \varphi_{i}}^{n}\left|\left[\left[\neg \varphi_{i}\right]\right]\right|-\sum_{i=1, \omega_{k} \not \models \varphi_{i}}^{n}\left|\varphi_{i}\right|$

We have $\forall i,\left|\varphi_{i}\right|=m-\left|\left[\llbracket \neg \varphi_{i}\right]\right|$, so we obtain:

$\beta_{k}\left(E_{\preceq^{d} D}\right)=\sum_{i=1, \omega_{k} \mid=\varphi_{i}}^{n}\left|\left[\neg \varphi_{i}\right]\right|-\sum_{i=1, \omega_{k} \mid \not \varphi_{i}}^{n}(m-$ $\mid\left[\left[\neg \varphi_{i}\right]||\right)$

$\left.=\quad \sum_{i=1, \omega_{k} \models \varphi_{i}}^{n} \mid \llbracket \neg \varphi_{i}\right]|| \quad \sum_{i=1, \omega_{k} \not \models \varphi_{i}}^{n} m+$ $\sum_{i=1, \omega_{k} \mid \neq \varphi_{i}}^{n}\left|\left[\left[\neg \varphi_{i}\right]\right]\right|$

$=\quad\left(\sum_{i=1, \omega_{k} \models \varphi_{i}}^{n} \mid\left[\left\lceil\neg \varphi_{i}\right]\left|+\sum_{i=1, \omega_{k} \not \varphi_{i}}^{n}\right|\left[\left\lceil\neg \varphi_{i}\right] \mid\right)-\right.\right.$

$\sum_{i=1, \omega_{k} \not \neq \varphi_{i}}^{n} m$

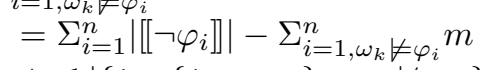

And $\left|\left\{i \in\{1, \ldots, n\}: \omega_{k} \mid \not \varphi_{i}\right\}\right|=$

Finally, we get:

$$
\sum_{i=1}^{n} d_{D}\left(\omega_{k}, \varphi_{i}\right)=d_{d_{D}, \Sigma}\left(\omega_{k}, E\right)
$$

$\beta_{k}\left(E_{\prec^{d_{D}}}\right)=\sum_{i=1}^{n} \mid\left[\left\lceil\neg \varphi_{i}\right]||-m * d_{d_{D}, \Sigma}\left(\omega_{k}, E\right)\right.$

Note that the term $\sum_{i=1}^{n}||\left[\neg \varphi_{i}\right]||$ is the same for all interpretations $\omega_{k}$, and that the multiplication by $-m$ reverses the scale, but does not change the relation given by the comparison of the distances $d_{d_{D}, \Sigma}\left(\omega_{k}, E\right)$.

So the preorders obtained by $\preceq_{d_{D}, \Sigma}$ and by the computation of the Borda scores $\beta$ (the pairwise comparisons) are the same. This completes the proof of Theorem 3 .

So the drastic merging operator is the only operator in the class of distance-based operators to be identical to its $\beta$ Borda version (i.e. to be a pairwise comparison operator). In the next section we will show a more general characterization of this drastic merging operator.

\section{Cancellation}

In this section we would like to introduce and discuss the notion of cancellation in belief merging.

In the characterization of the Borda rule, the cancellation property can be interpreted as requiring that a relation $a<b$ between two alternatives, can be cancelled by the converse relation $b<a$ (given by another individual). Please look at the formal definition of cancellation given in section 4 . 
We will now explore this general idea in order to define cancellation properties in belief merging. The idea is to state that a base in the profile can be cancelled by some other input. The exact definition of the "other input" will lead us to different cancellation properties.

Definition 11. A merging operator $\Delta$ satisfies the $(\neg \varphi$-Can $)$ property if for every formula $\varphi$, and every integrity constraint $\mu$,

$\left(\neg \varphi\right.$-Can) $\Delta_{\mu}(\varphi \sqcup \neg \varphi) \equiv \mu$

It is easy to verify the following:

Proposition 4. Let $\Delta$ be an IC merging operator. Then $\Delta$ satisfies $(\neg \varphi$-Can) if and only if it satisfies $(\neg E$-Can)

( $\neg E$-Can) $\Delta_{\mu}(E \sqcup \neg E) \equiv \mu$

Now we can provide a characterization of the drastic merging operator $\Delta^{d_{D}, f}$ in terms of cancellation :

Theorem 4. An IC merging operator $\Delta$ satisfies the $(\neg \varphi$ Can) cancellation property if and only if $\Delta=\Delta^{d_{D}, f}$.

Proof. To prove this theorem, we first prove the following proposition. We do not present this result as a lemma, because this proposition is interesting by itself and not only as a technical tool in a proof.

Proposition 5. The generating function $\triangleleft$ associated with an IC merging operator $\Delta$ generates only 2-strata preorders if and only if $\Delta=\Delta^{d_{D}, \Sigma}$.

Proof. $(\Rightarrow)$ Suppose that the generating function $\triangleleft$ associated with an IC merging operator $\Delta$ generates only 2-strata preorders.

Consider a profile $E$, and two interpretations $\omega_{1}$ and $\omega_{2}$. Suppose, without loss of generality, that the number of bases in $E$ entailed by $\omega_{1}$ is greater than the number of bases in $E$ entailed by $\omega_{2}$ (i.e. that $d_{d_{D}, \Sigma}\left(\omega_{1}, E\right)<d_{d_{D}, \Sigma}\left(\omega_{2}, E\right)$ ). To compare the two interpretations, we can distinguish and separate the bases into 4 cases:

Case 1: The bases $\varphi_{i}^{1}$ such that $\omega_{1} \models \varphi_{i}^{1}$ and $\omega_{2} \models \varphi_{i}^{1}$

Case 2: The bases $\varphi_{i}^{2}$ such that $\omega_{1} \not \models \varphi_{i}^{2}$ and $\omega_{2} \not \models \varphi_{i}^{2}$

Case 3: The bases $\varphi_{i_{1}}^{3}$ and $\varphi_{i_{2}}^{3}$, associated by pairs, such that $\omega_{1} \models \varphi_{i_{1}}^{3}, \omega_{2} \not \models \varphi_{i_{1}}^{3}$ and $\omega_{1} \not \models \varphi_{i_{2}}^{3}$ and $\omega_{2} \models \varphi_{i_{2}}^{3}$.

Case 4: The remaining bases $\varphi_{i}^{4}$, such that $\omega_{1} \models \varphi_{i}^{4}$ and $\omega_{2} \not \models \varphi_{i}^{4}$.

From (IC2), we know that $\omega_{1} \simeq_{\varphi_{i}^{1}} \omega_{2}$.

There are exactly two levels in the preorders associated with the $\varphi_{i}^{2}$. As $\omega_{1} \not \models \varphi_{i}^{2}$ and $\omega_{2} \not \models \varphi_{i}^{2}, \omega_{1}$ and $\omega_{2}$ belong to the same level for each $\preceq_{\varphi_{i}^{2}}$. In other words, $\forall \varphi_{i}^{2}, \omega_{1} \simeq_{\varphi_{i}^{2}}$ $\omega_{2}$. So $\omega_{1} \simeq \varphi_{i}^{2} \omega_{2}$.

For the case 3 , if $\omega_{1} \prec \varphi_{i_{1}}^{3} \sqcup \varphi_{i_{2}}^{3} \omega_{2}$ or $\omega_{1} \succ_{i_{1}}^{3} \sqcup \varphi_{i_{2}}^{3} \omega_{2}$, it contradicts (IC4). So $\omega_{1} \simeq_{\varphi_{i_{1}}^{3}} \sqcup \varphi_{i_{2}}^{3} \omega_{2}$.

For the case 4, it is clear that $\omega_{1} \prec_{\varphi_{i}^{4}} \omega_{2}$.

By (IC5) and (IC6), we get $\omega_{1} \simeq_{\varphi_{i}^{1} \sqcup \varphi_{i}^{2} \sqcup \varphi_{i_{1}}^{3} \sqcup \varphi_{i_{2}}^{3}} \omega_{2}$. As furthermore $\omega_{1} \prec_{\varphi_{i}^{4}} \omega_{2}$, we get $\omega_{1} \prec_{E} \omega_{2}$.

We just showed that if $\left.d_{d_{D}, \Sigma}\left(\omega_{1}, E\right)<d_{d_{D}, \Sigma}\left(\omega_{2}, E\right)\right)$, then $\omega_{1} \prec_{E} \omega_{2}$.
Through the same reasoning, we can show that if $\left.d_{d_{D}, \Sigma}\left(\omega_{1}, E\right)=d_{d_{D}, \Sigma}\left(\omega_{2}, E\right)\right)$, then $\omega_{1} \simeq_{E} \omega_{2}$. So the preorder obtained from $\Delta$ for $E$ coincides with the preorder obtained from $\Delta^{d_{D}, \Sigma}$.

$(\Leftarrow)$ This part is clear: if $\Delta=\Delta^{d_{D}, \Sigma}$, then the generating function $\triangleleft$ associated with $\Delta^{d_{D}, \Sigma}$ contains only 2-strata preorders.

An interesting corollary of Proposition 5 is the following one:

Corollary 1. If $\Delta^{d_{D}, f}$ is a merging operator then $\Delta^{d_{D}, f} \equiv$ $\Delta^{d_{D}, \Sigma}$.

Let us turn now to the proof of the main theorem. $\left(\Rightarrow:\right.$ ) Suppose that $\Delta \not \equiv \Delta^{d_{D}, f}$. Then, by proposition 5 , we know that there is at least one base $\varphi$ such that the assignment $\preceq_{\varphi}$ defines a preorder with more than two strata. So there are three interpretations $\omega_{1}, \omega_{2}$ and $\omega_{3}$ such that $\omega_{1} \models \varphi, \omega_{2} \not \models \varphi, \omega_{3} \not \models \varphi$ and $\omega_{2} \prec_{\varphi} \omega_{3}$.

We consider $\varphi^{\prime}=\neg \varphi$, and as $\Delta$ satisfies ( $\neg \varphi$-Can), $\Delta\left(\varphi \sqcup \varphi^{\prime}\right) \equiv \top$.

As $\omega_{2} \not \models \varphi$ and $\omega_{3} \not \forall \varphi$, we have that $\omega_{2} \models \varphi^{\prime}$ and $\omega_{3} \models \varphi^{\prime}$, so $\omega_{2} \simeq_{\varphi^{\prime}} \omega_{3}$.

From condition 6 of the syncretic assignment, as $\omega_{2} \prec_{\varphi}$ $\omega_{3}$ and $\omega_{2} \simeq_{\varphi^{\prime}} \omega_{3}$, then $\omega_{2} \prec\left\{\varphi, \varphi^{\prime}\right\} \omega_{3}$ : this contradicts the fact that $\Delta\left(\varphi \sqcup \varphi^{\prime}\right) \equiv \top$. So $\Delta \equiv \Delta^{d_{D}, f}$.

$\left(\Leftarrow\right.$ :) We have to prove that $\Delta^{d_{D}}, f$ satisfies the $(\neg \varphi$-Can $)$ property. First, we know from Corollary 1 that $\Delta^{d_{D}, f} \equiv$ $\Delta^{d_{D}, \Sigma}$. Now, we have to show that $\Delta^{d_{D}, \Sigma}$ satisfies the $(\neg \varphi$ Can) property. Consider a profile $E=\left\{\varphi_{1}, \ldots, \varphi_{n}\right\}$, its negation $\neg E=\left\{\neg \varphi_{1}, \ldots, \neg \varphi_{n}\right\}$ and an integrity constraint $\mu$.

Consider an interpretation $\omega$.

$\left.d^{d_{D}, \Sigma}(\omega, E)=\Sigma_{i=1}^{i=n} d_{D}\left(\omega, \varphi_{i}\right)\right)=k$, where $0 \leq k \leq n$.

$\left.d^{d_{D}, \Sigma}(\omega, \neg E)=\sum_{i=1}^{i=n} d_{D}\left(\omega, \neg \varphi_{i}\right)\right)=n-k$.

$d^{d_{D}, \Sigma}(\omega, E \sqcup \neg E)=k+(n-k)=n$.

As $\forall \omega, d^{d_{D}, \Sigma}(\omega, E \sqcup \neg E)=n$, all the interpretations of $\mu$ are equivalent and $\Delta_{\mu}(E \sqcup \neg E) \equiv \mu$.

In fact, after a careful reading of the proof, one can see that Theorem 4 may be expressed in a more general way. To make this Theorem true, we need:

- a generating function $\triangleleft$

- an assignment associated with the merging process, satisfying Condition 6 of the syncretic assignment.

We know that a distance $d$ between interpretations is a way to define a generating function, as it defines naturally a preorder on interpretations. So an interesting question arises about the expression of Theorem 4 when a distance $d$ and an aggregation function $f$ are available.

Proposition 6. If the aggregation function $f$ is anonymous and not decreasing, the merging operator $\Delta^{d, f}$ satisfies the $\left(\neg \varphi\right.$-Can) cancellation property if and only if $d=d_{D}$.

Proof. Suppose that a merging operator $\Delta^{d, f}$ satisfies the ( $\neg \varphi$-Can) property, and that $d \neq d_{D}$.

As $d \neq d_{D}$, there is at least one base $k$ and three interpretations $\omega_{1}, \omega_{2}, \omega_{3}$ such that $\omega_{1} \models k, \omega_{2} \not \models \varphi$, 
$d\left(\omega_{1}, \omega_{2}\right)$ is minimal among all the interpretations of $\neg \varphi$ (i.e. $\left.d\left(\omega_{1}, \omega_{2}\right)=\min _{\omega_{k} \models \neg \varphi}\left(d\left(\omega_{1}, \omega_{k}\right)\right)\right)$ and $d\left(\omega_{2}, \varphi\right)<$ $d\left(\omega_{3}, \varphi\right)$.

Consider $E=\left\langle\varphi_{\omega_{1}}, \varphi_{\neg \omega_{1}}\right\rangle$. By the ( $\neg$-Can) property, $\Delta\left(\left\langle\varphi_{\omega_{1}}, \varphi_{\neg \omega_{1}}\right\rangle\right) \equiv \top$, so $\omega_{3} \simeq_{E} \omega_{1}$.

But as $0=d\left(\omega_{1}, \varphi\right)<d\left(\omega_{2}, \varphi\right)<d\left(\omega_{3}, \varphi\right)$

And $0=d\left(\omega_{2}, \varphi_{\neg \omega_{1}}\right)=d\left(\omega_{3}, \varphi_{\neg \omega_{1}}\right)<d\left(\omega_{1}, \varphi\right)$.

As $d\left(\omega_{1}, \omega_{2}\right)=\min _{\omega_{k} \models \neg \varphi}\left(d\left(\omega_{1}, \omega_{k}\right)\right)=d\left(\omega_{1}, \varphi_{\neg \omega_{1}}\right)$, and $d\left(\omega_{1}, \omega_{2}\right)=d\left(\omega_{2}, \varphi_{\omega_{1}}\right)$, we have $d\left(\omega_{1}, \varphi_{\neg \omega_{1}}\right)=$ $d\left(\omega_{2}, \varphi_{\omega_{1}}\right)$.

As the aggregation function $f$ is not decreasing and anonymous, $d\left(\omega_{1},\left\{\varphi_{\omega_{1}}, \varphi_{\neg \omega_{1}}\right\}\right)=d\left(\omega_{2},\left\{\varphi_{\omega_{1}}, \varphi_{\neg \omega_{1}}\right\}\right)<$ $d\left(\omega_{3},\left\{\varphi_{\omega_{1}}, \varphi_{\neg \omega_{1}}\right\}\right)$

This contradicts $\Delta\left(\varphi_{\omega_{1}} \sqcup \varphi_{\neg \omega_{1}}\right) \equiv \top$.

We can stress that in Proposition 6, no assumption is made on the assignment associated with $\Delta^{d, f}$. In particular, we do not suppose that $\Delta^{d, f}$ is an IC merging operator.

The $(\neg \varphi$-Can) cancellation property is interesting for obtaining the above characterization, but it is a very particular instance of a larger family of cancellation properties that we would like to introduce and discuss now, and that could lead to other characterization results.

Definition 12 (Cancellation 1).

( $\varphi \varphi$-Can) $\forall \varphi \Delta_{\mu}(\varphi \sqcup \neg \varphi) \equiv \mu$

( $\varphi$-Can) $\forall \varphi \exists \varphi^{\prime} \Delta_{\mu}\left(\varphi \sqcup \varphi^{\prime}\right) \equiv \mu$

( $\bar{\varphi}$-Can) $\forall \varphi \Delta_{\mu}(\varphi \sqcup \bar{\varphi}) \equiv \mu$

We group here three related definitions, that share the fact that a base can be cancelled by another base. We begin by ( $\neg \varphi$-Can), which we already defined above. Note that this is a particular case, using negation, of the ( $\varphi$-Can) postulate, which states that any base can be cancelled by another base. Another potentially interesting particular case is $(\bar{\varphi}$ Can), where we use the dual of $\varphi$, as defined in (Haret and Woltran 2019), where $\bar{\varphi}$ is obtained by replacing every literal appearing in $\varphi$ by its negation.

But we can be a bit more general, and accept that maybe a single base will not be enough to cancel any base, and that a set of such bases will be necessary. This leads to other possible postulates :

Definition 13 (Cancellation 2).

(E-Can) $\forall \varphi \exists E \Delta_{\mu}(\varphi \sqcup E) \equiv \mu$

(fE-Can) $\forall \varphi \exists E$ s.t. $f(E)$ and $\Delta_{\mu}(\varphi \sqcup E) \equiv \mu$

( $\neg \omega$-Can) $\forall \varphi \exists E$ s.t. $\left(\forall \psi \in E \exists \omega_{i} \psi=\varphi_{\neg \omega_{i}}\right)$ and $\Delta_{\mu}(\varphi \sqcup E) \equiv \mu$

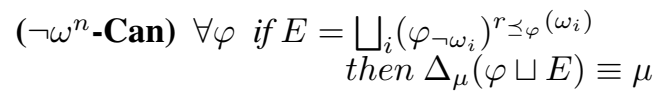

The most general form of these cancellation postulates is (E-Can) which states that some set of bases can cancel a chosen base. Then (fE-Can) is a restriction, where $E$ cannot be chosen freely, but must satisfy some condition $f()$. One such interesting restriction is given by $(\neg \omega$-Can) where all

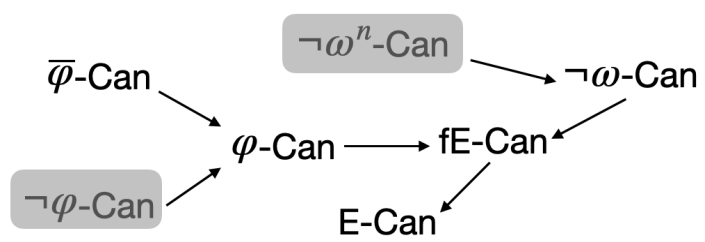

Figure 1: Cancellation postulates

the bases of $E$ have to be negations of a complete base $\varphi_{\omega}$. We further specialize this last postulate and obtain $\left(\neg \omega^{n}\right.$ Can) that gives the exact definition of the profile $E$ corresponding to a given base $\varphi$. This last form is related to $s$-Borda operators.

Let us note that all these postulates can be generalized for profiles not composed of a single base, but only in this work we treat these more simple forms. Let us just give the result for (E-Can) (but then notice that we can prove a similar result for all the other cancellation postulates):

Proposition 7. Let $\Delta$ be an IC merging operator. Then $\Delta$ satisfies $(\boldsymbol{E}-\boldsymbol{C a n})$ if and only if $\Delta$ satisfies $(\boldsymbol{E}-\boldsymbol{C a n E})$

(E-CanE) $\forall E^{\prime} \forall \varphi \exists E \Delta_{\mu}\left(E^{\prime} \sqcup \varphi \sqcup E\right) \equiv \Delta_{\mu}\left(E^{\prime}\right)$

Clearly some of the above postulates are more general than others :

Proposition 8. We have the relation of figure 1 , where $X \rightarrow$ $Y$ means that $Y$ is more general than $X$ (i.e. if $\Delta$ satisfies $X$ then it satisfies $Y$ ).

We let the exploration of the different forms of Cancellation for future work. We would just like to give a final result and a final claim.

Proposition 9. Let $\Delta$ be an operator that satisfies (IC1-IC3) and (IC5-IC8). If $\Delta$ is a s-Borda merging operator then $\Delta$ satisfies $\left(\neg \omega^{n}\right.$-Can).

Our claim is that we believe that $\left(\neg \omega^{n}\right.$-Can) encodes precisely the behaviour of $s$-Borda merging operators, but we do not have the formal proof yet.

So we put in grey in Figure 1 the two Cancellation postulates for which we have some links with concrete merging operators. We are convinced that further exploration of these links can provide us with other interesting characterizations of operators.

\section{Conclusion}

In this paper, we defined two families of merging operators inspired by the definition of the Borda voting rule. We also introduced the notion of cancellation in belief merging, inspired by the axiomatization of the Borda voting rule proposed by Young. This allowed us to show that the drastic merging operator is the only IC merging operator satisfying one variant of Cancellation, which gave us a characterization of this operator. We are convinced that a more systematic exploration of the other variants of Cancellation proposed by us (or other ones) can lead us to other interesting characterizations of belief merging operators. 


\section{Acknowledgements}

This work has benefited from the support of the AI Chair BE4musIA of the French National Research Agency (ANR). The fourth author has been partially funded by the program PAUSE of Collège de France.

\section{References}

Alchourrón, C. E.; Gärdenfors, P.; and Makinson, D. 1985. On the logic of theory change: Partial meet contraction and revision functions. Journal of Symbolic Logic 50:510-530.

Arrow, K.; Sen, A. K.; and Suzumura, K., eds. 2002. Handbook of Social Choice and Welfare, volume 1. NorthHolland.

Arrow, K. J. 1963. Social choice and individual values. New York: Wiley, second edition.

Borda, J. C. 1781. Mémoire sur les élections au srutin. Histoire de l'Académie Royale des Sciences.

Everaere, P.; Konieczny, S.; and Marquis, P. 2007. The strategy-proofness landscape of merging. Journal of Artificial Intelligence Research 28:49-105.

Everaere, P.; Konieczny, S.; and Marquis, P. 2010a. Disjunctive merging: Quota and gmin merging operators. Artificial Intelligence 174(12-13):824-849.

Everaere, P.; Konieczny, S.; and Marquis, P. 2010b. The epistemic view of belief merging: can we track the truth? In Nineteenth European Conference on Artificial Intelligence (ECAI'10), 621-626.

Everaere, P.; Konieczny, S.; and Marquis, P. 2014a. On egalitarian belief merging. In Fourteenth International Conference on Principles of Knowledge Representation and Reasoning (KR'14).

Everaere, P.; Konieczny, S.; and Marquis, P. 2014b. Counting votes for aggregating judgments. In International conference on Autonomous Agents and Multi-Agent Systems, (AAMAS'14), Paris, France, May 5-9, 2014, 1177-1184.

Everaere, P.; Konieczny, S.; and Marquis, P. 2015. Belief merging versus judgment aggregation. In Fourteenth International Conference on Autonomous Agents and Multiagent Systems (AAMAS'15), 999-1007.

Fermé, E., and Hansson, S. O. 2011. AGM 25 years: Twenty-five years of research in belief change. Journal of Philosophical Logic 40(2):295-331.

Gärdenfors, P. 1988. Knowledge in flux. MIT Press.

Haret, A., and Woltran, S. 2019. Belief revision operators with varying attitudes towards initial beliefs. In Proceedings of the Twenty-Eighth International Joint Conference on Artificial Intelligence (IJCAI'19), 1726-1733.

Katsuno, H., and Mendelzon, A. O. 1991. Propositional knowledge base revision and minimal change. Artificial Intelligence 52:263-294.

Konieczny, S., and Pino Pérez, R. 1998. On the logic of merging. In Sixth International Conference on Principles of Knowledge Representation and Reasoning (KR'98), 488498.
Konieczny, S., and Pino Pérez, R. 2002. Merging information under constraints: a logical framework. Journal of Logic and Computation 12(5):773-808.

Konieczny, S., and Pino Pérez, R. 2005. Propositional belief base merging or how to merge beliefs/goals coming from several sources and some links with social choice theory. European Journal of Operational Research 160(3):785802.

Konieczny, S., and Pino Pérez, R. 2011. Logic based merging. Journal of Philosophical Logic 40(2):239-270.

Konieczny, S.; Lang, J.; and Marquis, P. 2004. DA ${ }^{2}$ merging operators. Artificial Intelligence 157:49-79.

Lang, J.; Pigozzi, G.; Slavkovik, M.; van der Torre, L.; and Vesic, S. 2017. A partial taxonomy of judgment aggregation rules and their properties. Social Choice and Welfare 48(2):327-356.

Liberatore, P., and Schaerf, M. 1998. Arbitration (or how to merge knowledge bases). IEEE Transactions on Knowledge and Data Engineering 10(1):76-90.

Lin, J., and Mendelzon, A. O. 1998. Merging databases under constraints. International Journal of Cooperative Information System 7(1):55-76.

List, C., and Pettit, P. 2002. Aggregating sets of judgments. an impossibility result. Economics and Philosophy 18:89110.

List, C., and Puppe, C. 2009. Judgment aggregation: A survey. In The Handbook of Rational and Social Choice. Oxford University Press.

Mata Díaz, A., and Pino Pérez, R. 2017. Impossibility in belief merging. Artificial Intelligence 251:1 - 34 .

Merlin, V. 2003. The axiomatic characterizations of majority voting and scoring rules. Mathématiques et sciences humaines 41:87 - 109 .

Pigozzi, G. 2006. Belief merging and the discursive dilemma: an argument-based account to paradoxes of judgment aggregation. Synthese 152(2):285-298.

Revesz, P. Z. 1997. On the semantics of arbitration. International Journal of Algebra and Computation 7(2):133-160.

Young, H. P. 1974. An axiomatization of borda's rule. Journal of Economic Theory 9:43-52. 